\title{
A New Weight Scheme for the Shapley Value*
}

\author{
Guillaume Haeringer ${ }^{\dagger}$ \\ Universitat Autònoma de Barcelona
}

May 12, 2000

${ }^{*}$ I wish to thank Jean-Christophe Vergnaud for helpful discussions. I am much indebted to Francesc Carreras for his incisive and valuable comments. This work was completed while I was visiting the Insitute of Mathematical Economics at Bielefeld University. Financial Support from the European Commission is gratefully aknowledged. The usual disclaimer applies.

${ }^{\dagger}$ Departament d'Economia i Història Econòmica and CODE, Universitat Autònoma de Barcelona, 08193 Bellaterra, Spain, Tel: +34 935812505 Fax: +34 935812461 , E-mail: ghaeringer@volcano.uab.es 


\begin{abstract}
It is well known since Owen (1968) that the weights in the weighted Shapley value cannot be interpreted as a measure of power (i.e., of the ability to bargain) of the players. This paper proposes a new weight scheme for the Shapley value. Weights in this framework have to be interpreted as a measure of bargaining power. Two different axiomatic characterizations of this new value are proposed: one including the weights in the axioms and one without.
\end{abstract}

KEYWORDS Shapley value, monotonicity, weights.

Journal of Economic Literature Classification: C71 


\section{Introduction}

A cooperative game for some population of individuals describes the amount available to any subgroup of players. This field is sustained by two issues: the division problem and the formation of coalitions. This paper focuses on the first issue only. One of the most known solution concept for cooperative games is the Shapley value (Shapley 1953b). This value is based on four axioms: efficiency, dummy player, symmetry and additivity. ${ }^{1}$ In his Ph.D. thesis, Shapley extended his value to the non symmetric case. His motivation is as follows:

"It is easy to imagine games or game like situations in which the symmetry assumption is not appropriate, because of differences in the external characteristics of the players. For example [ ... ], there might be differences in bargaining ability."

This ability was introduced by Shapley by means of weights. To each player is assigned a weight, and the weighted Shapley value of some player in a unanimity game $u_{S}$ is the relative weight of that player in that coalition. Using the mathematical properties of the Shapley value, this weighted value was defined for any game - see Kalai and Samet $(1987,1988)$ for a general and complete characterization of the weighted Shapley value. Nevertheless, Owen (1968) brightly showed that weights cannot be interpreted as a measure of power, but rather as a measure of slowness to reach the grand coalition.

This paper proposes another way to introduce weights in the Shapley value, such that they can be interpreted as a measure of bargaining power. Our value is strongly based on the decomposition of games over the basis of unanimity games. Indeed, as shown by Shapley, any game can be decomposed as a linear combination of unanimity games. We argue that the coefficients of this decomposition provide some information about the relationships between the coalitions (and also the players) in the game. To make the point, consider any cooperative game $(N, v)$. Rewrite this game in terms of a linear combination of unanimity games. To each coalition $S \subseteq N$ corresponds then a unanimity game $u_{S}$ and a coefficient $\alpha_{S}$. This coefficient has been called by Harsanyi (1963) the dividend of the game for the coalition $S$. In other words, $\alpha_{S}$ is the part in $v(N)$ - the worth of the grand coalition - due to the formation of the subcoalition $S$. According to the Shapley value, players in $S$ have to share this amount $\alpha_{S}$. But this latter can be either positive or negative. If positive, then each player's value will be positive (restricted to the unanimity game $u_{S}$ ). Conversely, if $\alpha_{S}$ is negative, then each player will have to "pay," i.e., each player's value will be

\footnotetext{
${ }^{1}$ Shapley originaly proposed only three axioms: efficiency and dummy player were replaced by the carrier axiom.
} 
negative (again, restricted to the unanimity game $u_{S}$ ). It is our contention that the sharing rule cannot be the same for all cases $\left(\alpha_{S}\right.$ positive or negative). Indeed, when the coalition $S$ will have to share a positive amount of money, every player will try to maximize its profit. But when $\alpha_{S}<0$, the players will try to minimize their losses, i.e., to pay as less as possible. The modification we propose for the weight scheme is that for "negative" games, the order of plyers' power (i.e., of weights) must be inverted.

The paper is organized as follow. In section 2, we introduce the definitions and notations used throughout. We then provide in section 3 a complete characterization of our modified weighted Shapley value. Several axioms are introduced, and two existence theorems are stated: one including the weights in the axioms, and one without. Section 4 studies the monotonicity properties of our new value and section 5 is devoted to the proofs of the theorems.

\section{Definitions}

A game in characteristic function form is defined by a couple $(N, v)$ where $N$ is the set of players, $N=\{1, \ldots, n\}$, and $v$ is a mapping from the power set of $N$ to the set of real numbers: $v(S)$ designs the worth of the coalition $S \in 2^{N}$, with the convention $v(\varnothing) \equiv 0$. The space of all games with player set $N$ is denoted by $\Gamma_{N}$. Throughout the paper, capital latin letters will denote coalitions and their corresponding lower case will denote their cardinality (except $v$ which denotes the characterisitc function), e.g., $S$ is a coalition of size $s$. For convienance, we shall write $i$ instead of $\{i\}$. Set inclusion is supposed to be strict: $T \subset S$ means that $T$ is a subset of $S$ and that $T \neq S$. The sum of two games $v$ and $v^{\prime}$ is defined by $\left(v+v^{\prime}\right)(S)=v(S)+v^{\prime}(S)$ and the multiplication by a scalar $\lambda$ is defined by $(\lambda v)(S)=\lambda v(S)$. A unanimity game $u_{S}$ is a game such that $u_{S}(T)=1$ if $T \supseteq S$ and $u_{S}(T)=0$ otherwise. It is well known that the family of games $\left(u_{S}\right)_{S \subseteq N}$ forms a basis for $\Gamma_{N}$. Thus, any game can be decomposed as a linear combination of unanimity games: $v=\sum_{S \subseteq N} \alpha_{S} \cdot u_{S}$, where $\alpha_{S}=\sum_{T \subseteq S}(-1)^{s-t} v(T)$. Harsanyi (1963) called $\alpha_{S}$ the dividend of the game. A solution is a mapping $\phi: \Gamma_{N} \mapsto \mathbb{R}^{n}$, and its weighted counterpart is a map $\phi^{\omega}: \Gamma_{N} \times \mathbb{R}_{+} \mapsto \mathbb{R}^{n}$, where $\phi_{i}(v)$ denotes the value of player $i$ in the game $(N, v)$ with the solution $\phi$. We now introduce some definitions.

Definition 1 A game is positive (negative) if $\alpha_{S} \geq 0(\leq 0)$ for all $S \subseteq N$. A game is sign oriented if it is either positive or negative.

DEFINITION 2 Two games $v$ and $v^{\prime}$ are comparable if the coefficients of their decomposition in unanimity games are all of the same sign, i.e., $\alpha_{S}^{v} \cdot \alpha_{S}^{v^{\prime}} \geq 0, \forall S$. 
The Shapley value (Shapley 1953b) is the the solution $\varphi$ defined by

$$
\varphi_{i}(v)=\sum_{S \ni i} \alpha_{S} \cdot \varphi_{i}\left(u_{S}(S)\right)=\sum_{S \ni i} \alpha_{S} \cdot \frac{1}{s}, \quad \forall i \in N
$$

The weighted Shapley value is defined as follow (Shapley 1953a). For each unanimity games $u_{S}$ the weighted Shapley value $\varphi^{\omega}$ is:

$$
\varphi_{i}^{\omega}\left(u_{S}\right)= \begin{cases}\frac{\omega_{i}}{\omega_{S}} & \text { if } i \in S \\ 0 & \text { else }\end{cases}
$$

where $\omega_{S}=\sum_{j \in S} \omega_{j}$. It is obvious that if $\omega_{i}=\omega_{j}, \forall i, i \in N$, then $\varphi^{\omega}=\varphi$.

DEFINITION $3 \varphi^{\omega}$ is monotonic with respect to the weights if for any game $(N, v)$, for all player $i \in N$, one of this two statements is true:

1. $\frac{\partial \varphi_{i}^{\omega}(v)}{\partial \omega_{i}} \geq 0, \quad \forall \omega_{i} \in \mathbb{R}_{+}$,

2. $\frac{\partial \varphi_{i}^{\omega}(v)}{\partial \omega_{i}} \leq 0, \quad \forall \omega_{i} \in \mathbb{R}_{+}$,

Clearly, the weighted Shapley value is not monotonic with respect to the weights, as shown by Owen (1968). In the next section, we define a modified weighted Shapley value that is monotonic with respect to the weights, which allows us to understand weights as a measure of players' bargaining power.

\section{Modified weighted Shapley value}

In order to define the modified weighted Shapley value, we shall work with weight schemes. For a set of players $N$, we define a weight scheme as a vector $\bar{\omega}$ in $\mathbb{R}_{++}^{n}$, which describes the real bargaining power of agents. However, this number will be used differently, wether the amount to share is negative or positive.

Let $\bar{\omega}=\left(\bar{\omega}_{i}\right)_{i \in N} \in \mathbb{R}_{++}^{n}$. We define $\omega^{+}$and $\omega^{-}$as the bargaining power vector when players face a positive dividend and a negative dividend respectively,

$$
\begin{aligned}
\omega^{+} & =\left(\bar{\omega}_{1}, \ldots, \bar{\omega}_{n}\right), \\
\omega^{-} & =\left(\frac{1}{\bar{\omega}_{1}}, \ldots, \frac{1}{\bar{\omega}_{n}}\right) .
\end{aligned}
$$


The modified weighted Shapley value is the solution $\psi$ such that,

$$
\begin{gathered}
\psi_{i}^{\bar{\omega}}(v)=\sum_{S \ni i}\left|\alpha_{S}\right| \cdot \psi^{\bar{\omega}}\left(\operatorname{sgn}\left(\alpha_{S}\right) \cdot u_{S}\right)=\sum_{S \ni i} \alpha_{S} \cdot \omega_{i / S}^{v}, \\
\text { with } \omega_{i / S}^{v}= \begin{cases}\frac{\omega_{i}^{+}}{\sum_{j \in S} \omega_{j}^{+}} & \text {if } \alpha_{S} \geq 0, \\
\frac{\omega_{i}^{-}}{\sum_{j \in S} \omega_{j}^{-}} & \text {if } \alpha_{S}<0,\end{cases}
\end{gathered}
$$

where $\operatorname{sgn}\left(\alpha_{S}\right)$ is the sign of $\alpha_{S}$ defined by,

$$
\operatorname{sgn}\left(\alpha_{S}\right)=\left\{\begin{array}{l}
+1 \quad \text { if } \alpha_{S} \geq 0, \\
-1 \quad \text { if } \alpha_{S}<0 .
\end{array}\right.
$$

Any game can be decomposed as a linear combination of unanimity games. In some way, this decomposition reflects the attractions and the antagonisms in the game. Hence, $\alpha_{S}$ positive (negative) means that the formation of the coalition $S$ is beneficial (penalizing) for the grand coalition. In other words, if $\alpha_{S}>0\left(\alpha_{S}<0\right)$, then coalition $S$ contributes positively (negatively) to $v(N)$. Players have then to share the benefits of the game as well as the losses. For beneficial situations, players will try to maximize their share, whereas for other situations, the converse will hold. Tha is, for penalizing situations, agents will try to minimize their share. We now introduce the axioms that will characterize the modified weighted Shapley value.

Axiom 1 (EFficiency) $\sum_{i \in N} \phi_{i}(v)=v(N)$.

AXiom 2 (Dummy Player) If $i$ is a dummy player (i.e., $v(S \cup i)=v(S)$, $\forall S \subseteq N)$ then $\phi_{i}(v)=0$.

AXIOM 3 (SEmi-ADDitivity) if $v$ and $w$ are comparable, then $\phi(v+w)=$ $\phi(v)+\phi(w)$.

Axiom 4 ( $\bar{\omega}$-SYMmetrY) Let $(N, v)$ be a TU-game. Let $\pi$ be an automorphism of the game $(N, v)$. Then,

(i) If $(N, v)$ is positive, then,

$$
\bar{\omega}_{\pi(i)} \phi_{i}(v)=\bar{\omega}_{i} \phi_{\pi(i)}(v) .
$$

(ii) If $(N, v)$ is negative, then,

$$
\bar{\omega}_{i} \phi_{i}(v)=\bar{\omega}_{\pi(i)} \phi_{\pi(i)}(v) .
$$


Axiom 1 and 2 are usual. The main argument for semi additivity and axiom $\bar{\omega}$-symmetry is that a comparison between players or games can be done only when the games exhibit some kind of regularity conditions. In the $\bar{\omega}$-symmetry axiom, this comparison is not the same for positive and negative games, and following the semi-additivity axiom, tw games can be analyzed separatly only if their penalizing and beneficial coalitions are the same. Indeed, two different games may not have the same decomposition, and then will not treat coalitions (and then the players) equally. Axiom 3 is a weaker version of the additivity axiom. This latter states that two situations (i.e., two games) can be analyzed separatly. Axiom 3 narrows this to the case when the games exhibit the same characteristics about the antagonisms between the players. For instance, in some game $v$ forming coalition $S$ is penalizing $\left(\alpha_{S}^{v} \leq 0\right)$, and for another game $v^{\prime}$ the formation of same coalition is beneficial $\left(\alpha_{S}^{v^{\prime}}>0\right)$. Obviously, the game $w=v+v^{\prime}$ will hide some of the characteristics of the coalition $S$. The $\bar{\omega}$-symmetry is the usual $\omega$-symmetry, but applied to weight schemes, instead of simple weight vectors.

An equivalent way to express the $\bar{\omega}$-symmetry axiom is to say that in a negative unanimity game, the ratio of two players' value (hence the ratio of their weights) must be inverted with respect to the ratio of their value in a positive unanimity game. We now introduce our first result.

THEOREM 1 A solution $\phi$ with a weight scheme $\bar{\omega}$ satisfies the Efficiency axiom, the Null-player axiom, the Semi-additivity axiom, and the $\bar{\omega}$-symmetry axiom, if and only if $\phi$ is the modified weighted Shapley value $\psi(\cdot, \bar{\omega})$ defined in (4).

In this first characterization of the modified weighted Shapley value, the weight scheme is an exogenous data. In the remaining of the section, we also provide an axiomatic characteriation of the modified weighted Shapley value that does not mention the weight scheme in an explicit way. This approach has already been proposed by Kalai and Samet (1987) and Nowak and Radzik (1995).

Axiom 5 (Semi-Generalized Symmetry) Let $(N, v)$ and $\left(N, v^{\prime}\right)$ be two TU-games. Let $\pi$ be an automorphism of the games $(N, v)$ and $\left(N, v^{\prime}\right)$. Then,

(i) If $(N, v)$ and $\left(N, v^{\prime}\right)$ are positive, then,

$$
\phi_{i}(v) \phi_{\pi(i)}\left(v^{\prime}\right)=\phi_{\pi(i)}(v) \phi_{i}\left(v^{\prime}\right) .
$$

(ii) If $(N, v)$ and $\left(N, v^{\prime}\right)$ are negative, then,

$$
\phi_{i}(v) \phi_{i}\left(v^{\prime}\right)=\phi_{\pi(i)}(v) \phi_{\pi(i)}\left(v^{\prime}\right) .
$$


Semi-generalized symmetry is merely a generalization of $\bar{\omega}$-symmetry. The ratio between the two values of two symmetric players in two different games is kept unchanged, provided that the two games are of the same sign.

We have the following result.

TheOREM 2 A solution $\phi$ satisfies the Efficiency axiom, the Null player axiom, the Semi-additivity axiom, and the Semi-generalized symmetry axiom if and only if there is a weight scheme $\bar{\omega}$ such that $\phi$ is the modified weighted Shapley value defined in (4).

\section{Monotonicity}

Consider the weighted Shaley value of any player of some game $(N, v)$. We say that the value is monotonic if an increase of the weight of some player induces an increase in her value, given that the oponents' weights unchanged. Consider the following 3 players game: $v(i)=0, \forall i, v(12)=12, v(13)=$ $v(23)=6$ and $v(123)=12$. The decomposition in unanimity games is

$$
v(S)=12 \cdot u_{(12)}(S)+6 \cdot u_{(13)}(S)+6 \cdot u_{(23)}(S)-12 \cdot u_{(123)}(S)
$$

The weighted Shapley value with weight vector $\omega=(1,1,1)$ is $\varphi^{\omega}(v)=$ $(5,5,2)$. With $\omega^{\prime}=(1,1,3)$, one obtains: $\varphi^{\omega^{\prime}}(v)=(5.1,5.1,1.8)$. Clearly, the weighted Shapley value may not be monotonic, although the game is superadditive. Following Owen (1968), we know that weights in the (traditional) weighted Shapley value are a measure of the slowness to reach the grand coalition. It is easy to see that the paradox vanishes when the game is convex. Indeed, recall that a game is convex if

$$
v(S \cup i)-v(S) \leq v(T \cup i)-v(T), \forall S \subseteq T \subseteq N \backslash i .
$$

In words, a game is convex if players' marginal contributions are increasing with respect to the set inclusion. Moreover, it is well known that the (weighted) Shapley value is an average of a players' marginal contribution to all coalitions. Hence, if a player's weight increases, she will have a higher probability to join large coalitions than small ones, and thus will have a higher probability to have 'high' marginal contributions. It is worth to point out that $\alpha_{S}<0$ for some coalitions $S$ does not necessarily make the weighted Shapley value non-monotonic with respect to the weights. To make the point, consider the following 3 players game: $v(i)=0, \forall i, v(12)=12, v(13)=v(23)=4$ and $v(123)=18$. Clearly, this game is convex, which implies that the weighted Shapley value is monotone, ${ }^{2}$ although $\alpha_{(123)}$ is negative. The decomposition in unanimity games is

$$
v(S)=12 \cdot u_{(12)}(S)+4 \cdot u_{(13)}(S)+4 \cdot u_{(23)}(S)-2 \cdot u_{(123)}(S)
$$

\footnotetext{
${ }^{2}$ For a formal statement of this result, see Kalai and Samet (1987).
} 
On the contrary, our new weight scheme makes the weighted Shapley value beeing always monotonic, even for non-monotonic games. Yet, it is worth to point out that negative values can be obtained. Consider the same game as above, with $\bar{\omega}^{\prime \prime}=(1,2,3)$. The modified weighted Shapley value is: $\psi^{\bar{\omega}^{\prime \prime}}(v) \approx(-1.05,7.13,5.92)$. However, if players know that their value is not individually rationnal for some coalition, then the coalition will surely not form. This suggest that a complete analysis of values for cooperative games should not be separated from the study of coalition formation.

\section{Proofs of the theorems}

Proposition 1 A solution that satisfies the $\bar{\omega}-$ symetry axiom aslo satisifes the generalized symetry axiom.

Proof The proof is obvious and is left to the reader.

LEMMA 1

Let $i$ be a dummy player in the game $v$. Then $\alpha_{S}=0, \forall S \ni i .^{3}$

Proof Recall that if $i$ is dummy, then $v(S \cup i)=v(S) \forall S \subseteq N \backslash i$. We have:

$$
\begin{aligned}
& \alpha_{S}=v(S)+\sum_{T \subset S}(-1)^{s-t} v(T) \\
& =v(S \backslash i)-\sum_{\substack{T \subset S \\
T \mid t=s-1}} v(T)+\sum_{\substack{T \subset S \\
T \mid t=s-2}} v(T) \ldots-\sum_{j \in S} v(j) \\
& =v(S \backslash i)-\sum_{\substack{T \subset S \\
T \mid t=s-1 \\
i \notin T}} v(T)-\sum_{\substack{T \subset S \\
T \in t=s-1 \\
i \in T}} v(T)+\sum_{\substack{T \subset S \\
T \mid t=s-2 \\
i \notin T}} v(T)+\sum_{\substack{T \subset S \\
T \mid t=s-2 \\
i \in T}} v(T)-\ldots \\
& =v(S \backslash i)-v(S \backslash i)-\sum_{\substack{T \subset S \\
T \mid t=s-2 \\
i \notin T}} v(T)+\sum_{\substack{T \subset S \\
T \mid t=s-2 \\
i \notin T}} v(T)+\sum_{\substack{T \subset S \\
T \mid t=s-3 \\
i \notin T}} v(T) \\
& =\sum_{\substack{T \subset S \\
T \mid t=s-3 \\
i \notin T}} v(T)+\cdots=0
\end{aligned}
$$

\footnotetext{
${ }^{3}$ This lemma has already been stated by Kalai and Samet. However, their proof is different. They use an induction principle. They show that for a dummy player $i$, if $\alpha_{S}=0$ for all $s \leq k$, then $\alpha_{S}=0$ for $s=k+1$, for all $S \ni i$.
} 
LEMMA 2

Let $\phi$ be a solution that satisfies the dummy player, efficiency and either generalized symmetry or $\bar{\omega}-$ symmetry axioms. Then $\phi\left(\alpha_{S} u_{S}\right)=\left|\alpha_{S}\right| \phi\left(\operatorname{sgn}\left(\alpha_{S}\right) u_{S}\right)$

Proof By proposition 1, it suffices to prove the case for the generalized symmetry axiom. Suppose that $\alpha_{S}$ is positive. Take any player $i$ and $j$ in $S$ (The proof for $s=1$ is obvious). By generalized symmetry, we have: $\frac{\phi_{i}\left(u_{S}\right)}{\phi_{i}\left(\alpha_{S} u_{S}\right)}=\frac{\phi_{j}\left(u_{S}\right)}{\phi_{j}\left(\alpha_{S} u_{S}\right)}$, which yields $\phi_{i}\left(u_{S}\right)=\phi_{i}\left(\alpha_{S} u_{S}\right) \frac{\phi_{j}\left(u_{S}\right)}{\phi_{j}\left(\alpha_{S} u_{S}\right)}$. Sum over $i$. By efficiency, we get $1=\alpha_{S} \frac{\phi_{j}\left(u_{S}\right)}{\phi_{j}\left(\alpha_{S} u_{S}\right)} \Rightarrow \phi_{j}\left(\alpha_{S} u_{S}\right)=\alpha_{S} \phi_{j}\left(u_{S}\right)$.

If $\alpha_{S}<0$, then define $\beta_{S}:=-\alpha_{S}$. This yields: $\phi\left(\alpha_{S} u_{S}\right)=\phi\left(\beta_{S}-u_{S}\right)=$ $\beta_{S} \phi\left(-u_{S}\right)$ because $\beta_{S}>0$, which is tantamount to $\left|\alpha_{S}\right| \phi\left(\operatorname{sgn}\left(\alpha_{S}\right) u_{S}\right)$.

Proof of theorem 1 We first show that $\psi$ satisfies the efficiency, the semi-additivity, the $\bar{\omega}$-symmetry, and the null player axioms. Efficiency follows from the definition of $\psi$ :

$$
\begin{aligned}
\sum_{i \in N} \psi_{i}(v) & =\sum_{i \in N} \sum_{S \ni i} \alpha_{S} \frac{\omega_{i}}{\sum_{j \in S} \omega_{j}}=\sum_{S \subseteq N} \alpha_{S}\left(\sum_{i \in S} \frac{\omega_{i}}{\sum_{j \in S} \omega_{j}}\right) \\
& =\sum_{S \subseteq N} \alpha_{S} u_{S}(S)=v(N) .
\end{aligned}
$$

By lemma 1 applied to unanimity games, if $i$ is a null player, then $\alpha_{S}=$ $0, \forall S \ni i$. Then $\psi_{i}(v)=0$. Consider $v$ and $w$ such that $v$ and $w$ are comparable, then $\alpha_{S}^{v+w}$ is of same sign as $\alpha_{S}^{v}$ and $\alpha_{S}^{w}$. Let $N_{+}=\{S \subseteq$ $\left.N \mid \alpha_{S} \geq 0\right\}$ and $N_{-}=\left\{S \subseteq N \mid \alpha_{S}<0\right\}$.

$$
\begin{aligned}
\psi_{i}(v+w, \bar{\omega}) & =\sum_{S \ni i} \alpha_{S}^{v+w} \cdot \bar{\omega}_{i / S} v+w \\
& =\sum_{S \in N_{+}} \alpha_{S}^{v+w} \cdot \frac{\omega_{i}}{\sum_{j \in S} \omega_{j}}+\sum_{S \in N_{-}} \alpha_{S}^{v+w} \cdot \frac{\omega_{i}}{\sum_{j \in S} \omega_{j}} \\
& =\sum_{S \in N_{+}}\left(\alpha_{S}^{v}+\alpha_{S}^{w}\right) \cdot \frac{\omega_{i}^{+}}{\sum_{j \in S} \omega_{j}^{+}}+\sum_{S \in N_{-}}\left(\alpha_{S}^{v}+\alpha_{S}^{w}\right) \cdot \frac{\omega_{i}^{-}}{\sum_{j \in S} \omega_{j}^{-}} \\
& =\sum_{S \ni i} \alpha_{S}^{v} \bar{\omega}_{i / S} v+\sum_{S \ni i} \alpha_{S}^{w} \bar{\omega}_{i / S} w \\
& =\psi_{i}(v, \bar{\omega})+\psi_{i}(w, \bar{\omega}) .
\end{aligned}
$$

Thus $\psi$ satisfies the semi-additivity. Let us consider a positive game $(N, v)-$ 
the proof is analogous for a negative game. We have,

$$
\begin{aligned}
\psi_{i}(v, \bar{\omega}) & =\sum_{i \in S} \alpha_{S} \frac{\bar{\omega}_{i}}{\bar{\omega}_{S}} \\
& =\sum_{S \ni i} \alpha_{S} \cdot \frac{\omega_{i}^{+}}{\sum_{j \in S} \omega_{j}^{+}}=\frac{\omega_{i}^{+}}{\omega_{j}^{+}} \sum_{S \ni i} \alpha_{S} \cdot \frac{\omega_{j}^{+}}{\sum_{j \in S} \omega_{j}^{+}} \\
& =\frac{\omega_{i}^{+}}{\omega_{j}^{+}} \sum_{i \in S} \alpha_{S} \frac{\omega_{j}^{+}}{\omega_{S}^{+}}=\frac{\bar{\omega}_{i}}{\bar{\omega}_{j}} \psi_{j}(v, \bar{\omega}) .
\end{aligned}
$$

It remains to show that a solution $\phi$ satisfying the efficiency, the semiadditivity, the $\bar{\omega}$-symmetry, and the null player axioms is the modified weighted Shapley value $\psi(v, \bar{\omega})$. Let $\phi$ be a solution that satisfies these 4 axioms. Any game $(N, v)$ can be decomposed in a linear combination of unanimity games: $v=\sum_{S \subseteq N} \alpha_{S} u_{S}$. Let $v^{\prime}$ and $v^{\prime \prime}$ be defined by:

$$
\begin{aligned}
v^{\prime} & =\sum_{S \in N_{+}} \alpha_{S} u_{S}+\sum_{S \in N_{-}} 0 \cdot u_{S}, \\
v^{\prime \prime} & =\sum_{S \in N_{+}} 0 \cdot u_{S}+\sum_{S \in N_{-}} \alpha_{S} u_{S} .
\end{aligned}
$$

Clearly, $v^{\prime}$ and $v^{\prime \prime}$ are comparable. Moreover, $v^{\prime}$ (respectively $v^{\prime \prime}$ ) can be seen as a sum of $n_{+}$(respectively $n_{-}$) games: $v^{\prime}=\sum_{K \in N_{+}} v_{k}^{\prime}$, where $v_{k}^{\prime}=$ $\sum_{R \subseteq N} \alpha_{R} u_{R}$ with $\alpha_{R}=\alpha_{S}$ if $R=S$ and 0 otherwise. All the $v_{k}^{\prime}$ are comparable (they are all positive.) Thus $\phi\left(v^{\prime}\right)=\sum_{S \in N_{+}} \phi\left(\alpha_{S} u_{S}\right)$. The same property applies to $v^{\prime \prime}$. Because $\phi$ satisfies the null player axiom, it suffices to sum over the sets containing $i$, because $\phi_{i}\left(u_{S}\right)=0$ if $i \notin S$. By lemma $2, \phi_{i}(v)=\sum_{S \ni i} \phi_{i}\left(\alpha_{S}^{v} u_{S}\right)=\sum_{S \ni i}\left|\alpha_{S}^{v}\right| \phi_{i}\left(\operatorname{sgn}\left(\alpha_{S}\right) u_{S}\right)$. It remains to show that $\psi^{\omega}$ and $\phi$ coincide on each unanimity game. It is obvious that for all $S, u_{S}$ is of constant sign. Suppose first that $\alpha_{S} \geq 0$. Then by $\bar{\omega}$-symmetry, we have:

$$
\omega_{j} \phi_{i}\left(u_{S}\right)=\omega_{i} \phi_{j}\left(u_{S}\right)
$$

and

$$
\omega_{j} \psi_{i}\left(u_{S}, \bar{\omega}\right)=\omega_{i} \psi_{j}\left(u_{S}, \bar{\omega}\right) .
$$

Divide the first equality by the second:

$$
\frac{\phi_{i}\left(u_{S}\right)}{\psi_{i}\left(u_{S}, \bar{\omega}\right)}=\frac{\phi_{j}\left(u_{S}\right)}{\psi_{j}\left(u_{S}, \bar{\omega}\right)}=\lambda .
$$

Notice that $\lambda$ is constant, $\forall i, j \in S$. This implies that $\phi_{i}\left(u_{S}\right)=\lambda \psi_{i}\left(u_{S}, \bar{\omega}\right)$. Let sum over $S$, and because $\psi$ satisfies the efficiency and the null player 
axioms, we obtain:

$$
\sum_{i \in S} \phi_{i}\left(u_{S}\right)=\lambda \cdot \sum_{i \in S} \psi_{i}\left(u_{S}, \bar{\omega}\right)=1
$$

Thus $\lambda=1$, which implies that $\phi_{i}\left(u_{S}\right)=\psi_{i}\left(u_{S}, \bar{\omega}\right)$.

Suppose now that $\alpha_{S}<0$. Then, as $\psi$ and $\phi$ satisfies the $\bar{\omega}$-symmetry axiom, we obtain:

$$
\frac{\phi_{i}\left(u_{S}\right)}{\phi_{j}\left(u_{S}\right)}=\frac{\phi_{j}\left(-u_{S}\right)}{\phi_{i}\left(-u_{S}\right)}
$$

and

$$
\frac{\psi_{i}\left(u_{S}, \bar{\omega}\right)}{\psi_{j}\left(u_{S}, \bar{\omega}\right)}=\frac{\psi_{j}\left(-u_{S}, \bar{\omega}\right)}{\psi_{i}\left(-u_{S}, \bar{\omega}\right)}
$$

As $\phi_{i}\left(u_{S}\right)=\psi_{i}\left(u_{S}, \bar{\omega}\right), \forall i \in S, \forall S \subseteq N$, we obtain:

$$
\frac{\phi_{j}\left(-u_{S}\right)}{\phi_{i}\left(-u_{S}\right)}=\frac{\psi_{j}\left(-u_{S}, \bar{\omega}\right)}{\psi_{i}\left(-u_{S}, \bar{\omega}\right)} .
$$

Then

$$
\phi_{j}\left(-u_{S}\right)=\phi_{i}\left(-u_{S}\right) \frac{\psi_{j}\left(-u_{S}, \bar{\omega}\right)}{\psi_{i}\left(-u_{S}, \bar{\omega}\right)} .
$$

Sum over $j$, by the efficiency and the null player axiom, we obtain:

$$
\phi_{i}\left(-u_{S}\right)=\psi_{i}\left(-u_{S}, \bar{\omega}\right) .
$$

We now show that the $\bar{\omega}$-symmetry axiom implies the existence of two weight schemes, one for $\alpha_{S} \geq\left(\right.$ denoted $\omega_{i}^{+}$), and one for $\alpha_{S}<0$, (denoted $\left.\omega_{i}^{-}\right)$such that $\omega_{i}^{+}=1 / \omega_{i}^{-}, \forall i \in N$. If $\alpha_{S}<0$, then $\alpha_{S} \phi\left(u_{S}\right)=\alpha_{S} \phi\left(-u_{S}\right)$. By the power inversion axiom, we have:

$$
\frac{\phi_{i}\left(u_{S}\right)}{\phi_{j}\left(u_{S}\right)}=\frac{\phi_{j}\left(-u_{S}\right)}{\phi_{i}\left(-u_{S}\right)} .
$$

This implies that, using the efficiency axiom:

$$
\begin{aligned}
\sum_{j \in N} \phi_{j}\left(-u_{S}\right) & =\phi_{i}\left(u_{S}\right) \phi_{i}\left(-u_{S}\right)\left(\sum_{j \in S} \frac{1}{\phi_{j}\left(u_{S}\right)}\right)=1 \\
\Rightarrow \quad \phi_{i}\left(-u_{S}\right) & =\frac{1 / \phi_{j}\left(u_{S}\right)}{\sum_{i \in S} \frac{1}{\phi_{i}\left(u_{S}\right)}} \\
\Rightarrow \quad \sum_{j \in N} \omega_{j}^{-} & =\frac{1 / \omega_{i}^{+}}{\sum_{j \in N} 1 / \omega_{i}^{-}} .
\end{aligned}
$$


This holds true for all $i \in S, \forall S$. Then by identification, we have $\omega_{i}^{-}=$ $1 / \omega_{i}^{+}$. As $S$ was arbitrary, this complete the proof

Proof of theorem 2 By theorem 1, we only need to check that $\psi$ verifies the generalized symmetry axiom. But $\psi$ satisfies the $\bar{\omega}$-symmetry axiom. Thus $\psi$ also satisfies the semi-generalized symmetry axiom by proposition 1.

It remains to prove that a solution $\phi$ that satisfies the efficiency, dummy player, semi-additivity, and semi-generalized symmetry axioms is the modified weighted Shapley value $\psi^{\bar{\omega}}$ for some weight scheme $\bar{\omega}$. Most of this part of the proof is similar to the proof of theorem 1, except for the proof that $\psi^{\bar{\omega}}$ and $\phi$ coincide on positive unanimity games. ${ }^{4}$ By the generalized symmetry axiom applied to $\psi^{\bar{\omega}}$ and $\phi$, we get for some $i$ and $j$ both in $S$ and $T$ :

$$
\frac{\phi_{i}\left(u_{S}\right)}{\phi_{j}\left(u_{S}\right)}=\frac{\phi_{i}\left(u_{T}\right)}{\phi_{j}\left(u_{T}\right)}=\lambda
$$

and

$$
\frac{\psi_{i}\left(u_{S}, \bar{\omega}\right)}{\psi_{j}\left(u_{S}, \bar{\omega}\right)}=\frac{\psi_{i}\left(u_{T}, \bar{\omega}\right)}{\psi_{j}\left(u_{T}, \bar{\omega}\right)}=\mu
$$

This leads to:

$$
\phi_{i}\left(u_{S}\right)=\lambda \phi_{j}\left(u_{S}\right) \text { and }=\psi_{i}\left(u_{S}, \omega\right)=\mu \psi_{j}\left(u_{T}\right) .
$$

Using the efficiency and dummy player axioms, (both $\phi$ and $\psi$ satisfy these two axioms), we get:

$$
u_{S}(N)=\sum_{i \in N} \phi_{i}\left(u_{S}\right)=s \cdot \lambda \cdot \phi_{j}\left(u_{S}\right)
$$

and

$$
u_{S}(N)=\sum_{i \in N} \psi_{i}\left(u_{S}, \omega\right)=s \cdot \mu \cdot \psi_{j}\left(u_{S}, \bar{\omega}\right)
$$

This leads to:

$$
\lambda \cdot \phi_{j}\left(u_{S}\right)=\mu \cdot \psi_{j}\left(u_{S}, \bar{\omega}\right) .
$$

Let us sum over $j$ :

$$
\lambda u_{S}(N)=\mu u_{S}(N) .
$$

Then $\lambda=\mu$. Using equality (6), we obtain:

$$
\phi_{j}\left(u_{S}\right)=\psi_{j}\left(u_{S}, \bar{\omega}\right), \forall j \in N, \forall S \subseteq N .
$$

It suffices now to prove that $\phi$ and $\psi$ coincide again when the unanimity game is negative. This part of the proof is the same as in the proof of theorem 1, where the semi-generalized symmetry, efficiency and dummy players axioms are used - starting from equation $(* *)$.

\footnotetext{
${ }^{4}$ In the previous proof, we used the $\bar{\omega}$-symmetry axiom instead.
} 


\section{References}

Harsanyi, J. C. (1963): "A Simplified Bargaining Model for the $n$-Person Cooperative Game," International Economic Review, 4, 194-220.

Kalai, E., And D. SAmet (1987): "On weighted Shapley values," International Journal of Game Theory, 16, 205-222.

Kalai, E., And D. Samet (1988): "Weighted Shapley values," in The Shapley value, Essays in honor of L. Shapley, ed. by A. Roth, pp. 83-99. Cambridge Univ. Press, Cambridge.

Nowak, A. S., And T. RAdzIK (1995): "On Axiomatizations of the Weighted Shapley Values," Games and Economic Behavior, 8, 389-405.

Owen, G. (1968): "A Note on the Shapley Value," Management Science, $14,731-732$.

Shapley, L. S. (1953a): "Additive and Non-Additive Set Functions," Ph.D. thesis, Princeton University.

(1953b): "A Value for n-person Games," in Contributions to the Theory of Games, vol. 2, ed. by H. W. Kuhn, and A. Tucker, pp. 307317. Princeton University Press, Princeton, N. J., Annals of Mathematics Studies, no. 28. 